\title{
Tall Stature
}

National Cancer Institute

\section{Source}

National Cancer Institute. Tall Stature. NCI Thesaurus. Code C118691.

Height greater than two standard deviations above the mean of the appropriate reference population for the age and sex of the individual. 